\title{
SOME PROPERTIES OF CIRCLE MAPS WITH ZERO TOPOLOGICAL ENTROPY
}

\author{
YINI YANG
}

\begin{abstract}
Aвstract. For a circle map $f: \mathbb{S} \rightarrow \mathbb{S}$ with zero topological entropy, we show that a non-diagonal pair $\langle x, y\rangle \in \mathbb{S} \times \mathbb{S}$ is non-separable if and only if it is an IN-pair if and only if it is an IT-pair. We also show that if a circle map is topological null then the maximal pattern entropy of every open cover is of polynomial order.
\end{abstract}

\section{INTRODUCTION}

The study of the complexity of dynamical system is still an important topic nowadays. Different versions of chaos were proposed to represent the complexity in various senses. We refer the reader to the survey [16] and references therein for more aspects and details.

For interval maps, there are many known results. Here we want to name a few. In 1975, in their seminal work [17], Li and Yorke showed that if an interval maps has a periodic point with period 3 then it is chaotic. Since then, the phenomenon introduced in [17] is called Li-Yorke chaos. In 1986, Smital [21] showed that for an interval map with zero topological entropy, the map is Li-Yorke chaotic if and only if it has a non-separable pair. In 1989, Kuchta and Smital [11] showed that for a continuous interval map, one scrambled pair implies Li-Yorke chaos. In 1991, Franzova and Smital [4] showed that a interval map is Li-Yorke chaotic if and only if it has positive topological sequence entropy. We refer the reader to the book [19] for more details on this topic.

Some of the results for interval maps have been extended to circle maps or even graph maps. In 1990, Kuchta [10] showed that for a circle map, one scrambled pair implies Li-Yorke chaos. In 2000, Hirc [7] showed that a circle map is Li-Yorke chaotic if and only if it has positive topological sequence entropy. In 2014, Ruette and Snoha [20] showed that for a graph map, one scrambled pair implies Li-Yorke chaos. In 2017, Li, Oprocha, Yang and Zeng [14] showed that a graph map is Li-Yorke chaotic if and only if it has positive topological sequence entropy.

In the framework of so-called "local entropy theory", lots of notions were introduced to describe specific dynamical properties, see [5] for a recent survey. Among them there are IN-pairs and IT-pairs. In 2011, Li [13] proved that for an interval map with zero topological entropy, a non-diagonal pair is non-separable which is related to Li-Yorke chaos if and only if it is an IN-pair if and only if it is an IT-pair. In 2017, Li, Oprocha, Yang and Zeng [14] proved that a graph map is Li-Yorke chaotic if and only if it has an IN-pair if and only if it has an IT-pair. The authors in [14] also proposed an open question as follows.

2010 Mathematics Subject Classification. 37E10, 37B40, 54H20.

Key words and phrases. circle maps; non-separable pair; IN-pair; IT-pair; topological null; maximal pattern entropy. 
Question 1.1. Let $f: G \rightarrow G$ be a graph map with zero topological entropy. Is it true that: $\langle x, y\rangle$ is an IN-pair if and only if $\langle x, y\rangle$ is an IT-pair?

One of the main results of this paper is to answer this question for circle maps. Actually, we have the following theorem.

Theorem 1.2. Let $f: \mathbb{S} \rightarrow \mathbb{S}$ be a circle map with zero topological entropy and $x \neq y \in \mathbb{S}$. Then the following conditions are equivalent:

(1) $\langle x, y\rangle$ is a non-separable pair;

(2) $\langle x, y\rangle$ is an IT-pair;

(3) $\langle x, y\rangle$ is an IN-pair.

In 2009, Huang and Ye [8] proposed the concept of maximal pattern entropy. They proved that the maximal pattern entropy of a dynamical system is equivalent to the supremum of all topological sequence entropy. And they also proved that the maximal pattern entropy can take only discrete values. In [8], Huang and Ye conjectured that for each topological null system, the maximal pattern entropy is of polynomial order for every open cover. And they proved that the conjecture holds for a zero dimensional system. In 2011, Li [13] proved that the conjecture holds for interval maps.

Another main result of our paper is to show that the conjecture holds for circle maps by proving the following theorem.

Theorem 1.3. A circle map $f: \mathbb{S} \rightarrow \mathbb{S}$ is topological null if and only if $p_{\mathbb{S}, \mathcal{U}}^{*}$ is of polynomial order for any open cover $\mathcal{U}$ of $\mathbb{S}$.

The paper is organized as follows. In Section 2, we recall some basic notions of topological dynamical systems which will be used latter. In Section 3, several concepts and important lemmas related to circle maps are listed, We emphasize properties of the circle maps without periodic points and the structure of $\omega$-limit sets. In Section 4, we study the relationship of IN-pairs, IT-pairs and non-separable pairs, and then prove Theorem 1.2. In Section 5, we review some properties on maximal pattern entropy and prove Theorem 1.3 .

\section{Preliminaries}

Throughout this paper, let $\mathbb{N}, \mathbb{R}$ and $\mathbb{C}$ denote the set of all non-negative integers, real numbers, and complex numbers, respectively. Let $\mathbb{S}$ be the set $\{z \in \mathbb{C}:|z|=1\}$. Let $M$ denote a non-degenerate closed interval $I$ or the circle $\mathbb{S}$ and let $C(M, M)$ denote the set of all continuous maps of $M$ into itself. Denote by $\Delta$ all the diagonal pairs of $X^{2}$ where $X$ is a compact metric space. The length of an interval $I$ is denoted by $|I|$. The cardinality of a finite set $A$ is denoted by \# $(A)$. Denote by $\{a\}$ to take the fractional part of the number $a$.

Now we introduce some basic notions in topological dynamics. We start with topological dynamical system and then introduce some concepts, including $\omega$-limit set, periodic points, and eventually periodic points.

Definition 2.1. By a topological dynamical system, we mean a pair $(X, f)$, where $X$ is a compact metric space with a metric $d$ and $f$ is a continuous map.

Definition 2.2. Let $(X, f)$ be a topological dynamical system. A point $x \in X$ is called periodic (denoted by $x \in \operatorname{Per}(f)$ ) with period $n$ if $f^{n}(x)=x$ and $f^{i}(x) \neq x$ for any 
$1 \leq i<n$. The set of periods of $f$ is denoted by $P(f)$ that is if $x \in \operatorname{Per}(f)$ has period $n$ then $n \in P(f)$. A periodic point with period 1 is called a fixed point (denoted by $x \in \operatorname{Fix}(f))$.

Definition 2.3. Let $(X, f)$ be a topological dynamical system and $x \in X$. We define the $\omega$-limit set of $x$ as

$$
\omega(x, f)=\bigcap_{n \geq 1} \overline{\left\{f^{i}(x): i \geq n\right\}}
$$

and the $\omega$-limit set of $f$ as

$$
\omega(f)=\bigcup_{x \in X} \omega(x, f)
$$

Definition 2.4. Let $(X, f)$ and $(Y, g)$ be two topological dynamical systems. A continuous map $\pi: X \rightarrow Y$ is a semi-conjugacy between $f$ and $g$ if $\pi$ is onto and $\pi \circ f=g \circ \pi$. If in addition $\pi$ is a homeomorphism, then $\pi$ is called a conjugacy between $f$ and $g$.

Definition 2.5. Let $X$ be a compact metric space and $C_{X}^{o}$ be the set of open covers of $X$. For $\mathcal{U} \in C_{X}^{o}$, we define $N(\mathcal{U})$ as the minimum cardinality of subcovers of $\mathcal{U}$. The join of two open covers $\mathcal{U}, \mathcal{V} \in C_{X}^{o}$ is

$$
\mathcal{U} \vee \mathcal{V}=\{U \cap V: U \in \mathcal{U}, V \in \mathcal{V}\}
$$

Definition 2.6. Let $(X, f)$ be a topological dynamical system and $\mathcal{U}, \mathcal{V} \in C_{X}^{o}$. The topological entropy of $f$ with respect to $\mathcal{U}$ is defined as

$$
h_{\text {top }}(f, \mathcal{U})=\lim _{n \rightarrow \infty} \frac{1}{n} \log \left(N\left(\bigvee_{i=1}^{n} f^{-i}(\mathcal{U})\right)\right)
$$

and the topological entropy of $(X, f)$ is defined as

$$
h_{\text {top }}(f)=\sup _{\mathcal{U} \in C_{X}^{o}} h_{\text {top }}(f, \mathcal{U})
$$

Definition 2.7. Let $(X, f)$ be a topological dynamical system and $A=\left\{a_{1}<a_{2}<a_{3}<\cdots\right\}$ an increasing sequence of positive integers. Take $\mathcal{U} \in C_{X}^{o}$. The topological sequence entropy of $f$ with respect to $\mathcal{U}$ along the sequence $A$ is defined as

$$
h_{\text {top }}^{A}(f, \mathcal{U})=\lim _{n \rightarrow \infty} \frac{1}{n} \log \left(N\left(\bigvee_{i=1}^{n} f^{-a_{i}}(\mathcal{U})\right)\right),
$$

the topological sequence entropy of $(X, f)$ along the sequence $A$ is defined as

$$
h_{\mathrm{top}}^{A}(f)=\sup _{\mathcal{U} \in C_{X}^{o}} h_{\mathrm{top}}^{A}(f, \mathcal{U}) .
$$

and the topological sequence entropy of $(X, f)$ is

$$
h_{\text {top }}^{\infty}(f)=\sup _{A} h_{\text {top }}^{A}(f),
$$

where the supreme takes over all increasing sequences of positive integers.

Moreover, $(X, f)$ is called topological null if $h_{\text {top }}^{\infty}(f)=0$ and otherwise it is called non-null.

The following two lemmas can be verified easily by the definition of topological semiconjugacy. 
Lemma 2.8. Let $(X, f)$ and $(Y, g)$ be two topological dynamical systems and $\pi: X \rightarrow Y$ is $a$ semi-conjugacy between $f$ and $g$. It holds that for any $x \in X, \pi(\omega(x, f))=\omega(\pi(x), g)$.

Lemma 2.9. Let $\varphi:(X, f) \rightarrow(Y, g)$ be a topological semi-conjugacy. Then $h_{\mathrm{top}}(g) \leq$ $h_{\text {top }}(f)$ and $h_{\text {top }}^{\infty}(g) \leq h_{\text {top }}^{\infty}(f)$.

Definition 2.10. Let $(X, f)$ be a topological dynamical system and $A_{1}, A_{2}, \cdots, A_{k} \subset X$. We call $I \subset \mathbb{N}$ an independence set of $\left\{A_{1}, A_{2}, \cdots, A_{k}\right\}$ if for any non-empty finite subset $J$ of $I$ and $S \in\{1,2, \ldots, k\}^{J}, \bigcap_{i \in J} f^{-i} A_{S(i)} \neq \emptyset$.

Definition 2.11. Let $(X, f)$ be a topological dynamical system. A pair $\langle x, y\rangle \in X \times X$ is called an IN-pair (reps. an IT-pair) if for any neighborhoods $U_{1}$ and $U_{2}$ of $x$ and $y$ respectively, $\left\{U_{1}, U_{2}\right\}$ has arbitrarily large finite independence sets (reps. $\left\{U_{1}, U_{2}\right\}$ has an infinite independence set). Denote the set of IN-pairs and IT-pairs of $(X, f)$ by $\operatorname{IN}(X, f)$ and $I T(X, f)$ respectively.

It is clear that every IT-pair is also an IN-pair.

Theorem 2.12 ([9]). A topological dynamical system is topological null if and only if every IN-pair is diagonal.

Lemma 2.13 ([9]). Let $(X, f)$ and $(Y, g)$ be two topological dynamical systems and $\pi: X \rightarrow$ $Y$ is a semi-conjugacy between $f$ and $g$. Then $\pi \times \pi(I N(X, f))=\operatorname{IN}(Y, g)$ and $\pi \times$ $\pi(I T(X, f))=I T(Y, g)$.

The following is a "folklore" result, see e.g. [22, Theorem 2.6] but without proofs. Here we provide a proof for completeness.

Lemma 2.14. Let $(X, f)$ be a topological dynamical system. Then for every $p \in \mathbb{N}$, $I N(X, f)=I N\left(X, f^{p}\right)$ and $I T(X, f)=I T\left(X, f^{p}\right)$.

Proof. We only prove the case $I N(X, f)=I N\left(X, f^{p}\right)$, as the case $I T(X, f)=I T\left(X, f^{p}\right)$ is similar.

$(\Rightarrow)$ Let $\langle x, y\rangle$ be an IN-pair of $(X, f)$. By the definition, for any neighborhoods $U_{1}$ of $x$ and $U_{2}$ of $y$ respectively and any $m \in \mathbb{N},\left\{U_{1}, U_{2}\right\}$ has an independence set $I$ with length $m p$, since the number of the remainders of $\bmod p$ is finite, if we denote $Q_{r}$ as the set of all numbers of the independece set $I$ which $\bmod p$ has the remainder $r$, there exist at least a $r \in\{0,1, \cdots, p-1\}$ such that the number of $Q_{r}$ is larger than or equal to $m$. We choose one $r$ which satisfies this condition and choose $m$ elements $\left\{q_{1}, q_{2}, \cdots, q_{m}\right\}$ from $Q_{r}$. Denote $I_{m}=\left\{l_{1}, l_{2}, \cdots, l_{m}\right\}$ such that for all $i \in\{1,2, \cdots, m\}, q_{i}=l_{i}+r$. Now we will show that $I_{m}$ is an independence set of $\left\{U_{1}, U_{2}\right\}$ under $f^{p}$ which has length $m$. For all $J_{m} \subset I_{m}$ which $J_{m}$ is a finite set, there exists a finite set $J \subset I$ such that $J=p \cdot J_{m}+r$. For any $\sigma=\{1,2\}^{J_{m}}$,

$$
f^{-r}\left(\bigcap_{i \in J_{m}}\left(f^{p}\right)^{-i} U_{\sigma(i)}\right)=\bigcap_{i \in J_{m}} f^{-r}\left(f^{-p i} U_{\sigma(i)}\right)=\bigcap_{i \in J_{m}} f^{-r-p i} U_{\sigma(i)}=\bigcap_{j \in J} f^{-j} U_{\sigma(i)} .
$$

Take $\sigma^{\prime}=\{1,2\}^{J}$ such that for all $j=r+p i \in J, \sigma^{\prime}(j)=\sigma(i)$. Since $I$ is an independence set of $f$,

$$
\bigcap_{j \in J} f^{-j} U_{\sigma^{\prime}(j)}=\bigcap_{\substack{j \in J \\ 4}} f^{-j} U_{\sigma(i)} \neq \emptyset .
$$


Therefore $f^{-r}\left(\bigcap_{i \in J_{m}}\left(f^{p}\right)^{-i} U_{\sigma(i)}\right) \neq \emptyset$ and $\bigcap_{i \in J_{m}}\left(f^{p}\right)^{-i} U_{\sigma(i)} \neq \emptyset$ obviously. Thus $\langle x, y\rangle$ is an IN-pair of $\left(X, f^{p}\right)$.

$(\Leftarrow)$ Let $\langle x, y\rangle$ be an IN-pair of $\left(X, f^{p}\right)$. By the definition, for any neighborhoods $U_{1}$ of $x$ and $U_{2}$ of $y$ respectively and any $m \in \mathbb{N}$, there exists an independence set $I_{m}$ of $\left\{U_{1}, U_{2}\right\}$ under $f^{p}$ with length $m$. Now we will claim that $I_{m}^{\prime}=p I_{m}$ is an independence set of $\left\{U_{1}, U_{2}\right\}$ under $f$ with length $m$. For all $J_{m}^{\prime} \subset I_{m}^{\prime}$, there exists $J_{m} \subset I_{m}$ such that $J_{m}^{\prime}=p J_{m}$. For any $\sigma^{\prime}=\{1,2\}^{J_{m}^{\prime}}$, there exists $\sigma=\{1,2\}^{J_{m}}$ such that for all $j=p i \in J_{m}^{\prime}$, $\sigma^{\prime}(j)=\sigma(i)$. Then

$$
\bigcap_{j \in J_{m}^{\prime}} f^{-j} U_{\sigma^{\prime}(j)}=\bigcap_{i \in J_{m}} f^{-p i} U_{\sigma(i)}=\bigcap_{i \in J_{m}}\left(f^{p}\right)^{-i} U_{\sigma(i)} \neq \emptyset .
$$

Hence $\langle x, y\rangle$ is an IN-pair of $f$.

\section{Preparations on circle maps}

3.1. General notions. In this subsection we introduce natural projection, the ordering and metric of the circle, and orientation-preserving homeomorphism. Besides we introduce the definition of lifting, degree and rotation number of the circle. We refer the readers to the textbook [1] and [6] for more informations.

Definition 3.1. The natural projection $e: \mathbb{R} \rightarrow \mathbb{S}$ is the map $e(x)=\exp (2 \pi i x)$.

According to the natural projection, we also view the circle $\mathbb{S}$ as $[0,1)(\bmod 1)$.

Definition 3.2. We define a metric $d$ on $\mathbb{S}$ as follows: for any $x, y \in \mathbb{S}$,

$$
d(x, y)=\min \left\{\left|x^{\prime}-y^{\prime}\right|: e\left(x^{\prime}\right)=x, e\left(y^{\prime}\right)=y\right\} .
$$

It is easy to see that the distance between $a$ and $b$ can be described as follows

$$
d(a, b)=\left\{\begin{array}{l}
|a-b|, \quad|a-b| \leq \frac{1}{2}, \\
1-|a-b|, \quad|a-b|>\frac{1}{2} .
\end{array}\right.
$$

Definition 3.3. Given $x_{0}, x_{1}, x_{2}, \cdots, x_{n} \in \mathbb{S}$, take $\tilde{x}_{0}, \tilde{x}_{1}, \tilde{x}_{2}, \cdots, \tilde{x}_{n} \in\left[\tilde{x}_{0}, \tilde{x}_{0}+1\right) \subset \mathbb{R}$ such that $e\left(\tilde{x}_{i}\right)=x_{i}$, the ordering of $\left(x_{0}, x_{1}, x_{2}, \cdots, x_{n}\right)$ on $\mathbb{S}$ is the permutation of $\{1,2, \cdots, n\}$ which satisfies that $\tilde{x}_{0}<\tilde{x}_{\sigma_{(1)}}<\tilde{x}_{\sigma_{(2)}}, \cdots<\tilde{x}_{\sigma_{(n)}}<\tilde{x}_{0}+1$.

Definition 3.4. A circle map $f \in C(\mathbb{S}, \mathbb{S})$ is called orientation-preserving homeomorphism if $f$ is a homeomorphism and it preserves the ordering which is defined in Definition 3.3 under $f$.

The following theorem is well known, see the textbook [6] for more detail.

Theorem 3.5. Let $f \in C(\mathbb{S}, \mathbb{S})$. We call a continuous function $F: \mathbb{R} \rightarrow \mathbb{R}$ a lifting of $f$ if $e \circ F=f \circ e$. The integer $d \in \mathbb{Z}$ such that $F(x+1)=F(x)+d$ exists for all $x \in \mathbb{R}$ and is called the degree of $f$ (or $F)$, denoted by $\operatorname{deg}(f)$.

In later parts of this paper, we denote by $\mathcal{L}_{1}$ the set of all liftings of the circle maps with $\operatorname{deg}(f)=1$.

Definition 3.6. Let $F \in \mathcal{L}_{1}$ and $x \in \mathbb{R}$. We define $\bar{\rho}(x)$ and $\underline{\rho}(x)$ as follows :

$$
\bar{\rho}(x)=\limsup _{n \rightarrow \infty} \frac{F^{n}(x)-x}{n} \text { and } \underline{\rho}(x)=\liminf _{n \rightarrow \infty} \frac{F^{n}(x)-x}{n}
$$


When $\bar{\rho}(x)=\rho(x)$ we write only $\rho(x)$. The number $\rho(x)$ is called the rotation number of $x$ with respect to $F$.

The following lemma is classical, see e.g. [1, Chapter III, Section 7] for details.

Lemma 3.7. Let $F \in \mathcal{L}_{1}$ and be a non-decreasing map. Then for any $x \in \mathbb{R}$, the limit

$$
\lim _{n \rightarrow \infty} \frac{F^{n}(x)-x}{n}
$$

exists and is independent of $x$. Therefore in the later parts of our paper, we can define $\rho(F)$ as $\rho_{F}(x)$ for any $x \in \mathbb{R}$. Moreover, the limit is a rational number if and only if $f$ has periodic points.

Remark 3.8. Let $f$ be an orientation preserving homeomorphism. It is easy to see that for any two liftings $F_{1}$ and $F_{2}$ of $f, \rho\left(F_{1}\right)-\rho\left(F_{2}\right) \in \mathbb{Z}$. Therefore we can define $\rho(f)=\{\rho(F)\}$ for any lifting $F$.

Lemma 3.9 ([1, Lemma 4.7.1]). Let $f \in C(\mathbb{S}, \mathbb{S})$ and $F$ be a lifting of $f$. Assunme that there exists a closed interval I invariant for $F$. Then $h_{\mathrm{top}}\left(\left.F\right|_{I}\right) \leq h_{\mathrm{top}}(f)$.

Remark 3.10. If $|I| \geq 1$, by lemma 2.9, we get the conclusion that $h_{\text {top }}\left(\left.F\right|_{I}\right)=h_{\text {top }}(f)$

In order to describe the properties of the periodic points of interval maps and circle maps, we introduce the following definition.

Definition 3.11. Let $\mathbb{N}_{s h}=\mathbb{N} \cup\left\{2^{\infty}\right\}$. We define an order of the numbers in $\mathbb{N}_{s h}$ as follow:

$$
\begin{aligned}
& 3>5>7>\cdots \\
& >2 \cdot 3>2 \cdot 5>2 \cdot 7>\cdots \\
& >2^{2} \cdot 3>2^{2} \cdot 5>2^{2} \cdot 7>\cdots \\
& \cdots \\
& >2^{\infty}>\cdots>2^{n}>\cdots>4>2>1,
\end{aligned}
$$

and call $\left(\mathbb{N}_{s h},>\right)$ Sharkovsky order.

Let $a \in \mathbb{N}_{\text {sh }}$ and denote $S(a)=\left\{m \in \mathbb{N}_{s h}: a \geq m\right\}$.

The following result is the well-known Sharkovsky theorem.

Theorem 3.12 (see e.g. [1]). Let $f \in C(I, I)$, then there exists $n \in \mathbb{N}_{\text {sh }}$ such that $P(f)=$ $S(n)$.

For interval maps with zero topological entropy, we characterize the properties of periodic points as follow.

Theorem 3.13 (see e.g. [1]). Let $f \in C(I, I)$ with $h_{\text {top }}(f)=0$. Then there exists $n \in \mathbb{N}_{\text {sh }}$ such that $2^{\infty} \geq n$ and $P(f)=S(n)$.

The properties of periodic points of circle maps are more complicated, we just consider the case of circle maps with zero topological entropy.

In the following theorem, see Theorem 3.5.3 and Theorem 3.6.8 of the textbook[1] when $\operatorname{deg}(f)=0$ and $\operatorname{deg}(f)=-1 \operatorname{deg}(f)=-1$. About the case when $\operatorname{deg}(f)=1$, we refer the readers to Corollary 3.9.7 and Theorem 3.10.1. 
Theorem 3.14. Let $f \in C(\mathbb{S}, \mathbb{S})$ with $|\operatorname{deg}(f)| \leq 1, h_{\text {top }}(f)=0$ and $\operatorname{Per}(f) \neq \emptyset$. Then there exist $k \in \mathbb{N}$ and $n \in \mathbb{N}_{\text {sh }}$ such that $2^{\infty} \geq n$ and $P(f)=k S(n)$. Moreover if $\operatorname{deg}(f)=0$ or $\operatorname{deg}(f)=-1$, then $f$ has a fixed point, therefore $k=1$.

Remark 3.15. Let $f \in C(\mathbb{S}, \mathbb{S})$ with $|\operatorname{deg}(f)| \leq 1$ and $h_{\text {top }}(f)=0$. By Lemma3.14, there exist $k \in \mathbb{N}$ and $n \in \mathbb{N}_{s h}$ such that $2^{\infty} \geq n$ and $P(f)=k S(n)$. In other words the minimal period of the periodic points of $f$ is $k$, and the other periods are all multiple of $k$. If $J \subset \mathbb{S}$ is a periodic interval with period $m>1$, then there exists $x \in J$ such that $x$ is a perodic point with period $m$. Thus $m$ is also the multiple of $k$.

3.2. Circle maps without periodic points. In this subsection we recall some properties of the circle maps without periodic points.

Definition 3.16. Let $\alpha \in[0,1)$ and $R_{\alpha}:[0,1) \rightarrow[0,1), x \rightarrow x+\alpha(\bmod 1)$. We call $R_{\alpha}$ the rotation of the circle with rotation number $\alpha$.

Lemma 3.17 ([2], see also [6, Section 4.1]). Let $f \in C(\mathbb{S}, \mathbb{S})$ without periodic points. Assume that $f$ is a homeomorphism. Then for any $x, y \in \mathbb{S}, \omega(x, f)=\omega(y, f)$. Denote $W=\omega(x, f)$ and $\rho(f)$ the rotation number of $f, \rho(f) \notin \mathbb{Q}$ since $f$ has no periodic points. In this case $W$ is a perfect set and exactly one of the following alternatives holds :

(1) if $f$ is topological transitive, $W=\mathbb{S}$ and $f$ topological conjugate to $R_{\rho(f)}$.

(2) if $f$ is not topological transitive, $W$ is a nowhere dense set and $f$ topological semi-conjugate to $R_{\rho(f)}$.

Lemma $3.18([2])$. Let $f \in C(\mathbb{S}, \mathbb{S})$ without periodic points. Assume that $f$ is not a homeomorphism. Then for any $x, y \in \mathbb{S}, \omega(x, f)=\omega(y, f)$. Denote $W=\omega(x, f)$. Then $W$ is a nowhere dense perfect set. Moreover, $\left.f\right|_{W}$ is orientation preserving or orientation reserving.

Lemma 3.19 ([7], Theorem 1]). Let $f \in C(\mathbb{S}, \mathbb{S})$. Then $f$ is not Li-Yorke chaotic if and only if $f$ is topological null. In particular if $f$ has no periodic points, then $f$ is topological null.

\subsection{The structure of $\omega$-limit sets of circle maps with zero topological entropy.}

Definition 3.20. Let $f \in C(M, M)$. A closed subinterval $J$ of $M$ is called periodic if there exists a positive integer $n$ such that $J, f(J), \ldots, f^{n-1}(J)$ are pairwise disjoint and $f^{n}(J)=J$. In this case, $n$ is the period of $J$ and $\left\{f^{i}(J)\right\}_{i=1}^{n-1}$ is called a cycle of intervals.

The following result is due to Sharkovsky, see e.g. Proposition 5.23 and Remarks on graph maps in page 132 of [19].

Lemma 3.21. Let $f \in C(M, M)$ with $h_{\text {top }}(f)=0$. For any $x \in M$, either $\omega(x, f)$ is a periodic orbit or $\omega(x, f)$ is infinite and $\omega(x, f) \cap \operatorname{Per}(f)=\emptyset$.

The following result can be easily confirmed by the definition.

Lemma 3.22. Let $f \in C(M, M)$ with $h_{\text {top }}(f)=0$ and $J$ be a periodic subinterval of $M$ with period $n$. For any $x \in M$, either $\omega(x, f) \subset \bigcup_{i=0}^{n-1} f^{i}(J)$ or $\omega(x, f) \cap \bigcup_{i=0}^{n-1} f^{i}(J)=\emptyset$.

We will need the following structure of $\omega$-limit sets of interval maps and circle maps. 
Lemma 3.23 ([3]). Let $f \in C(M, M)$ with $h_{\text {top }}(f)=0$ and $\operatorname{Per}(f) \neq \emptyset$. For any $x \in M$, if $\omega(x, f)$ is infinite, then there is a period portion of the $\omega$-limit set $\omega(x, f)$, i.e. there exists a sequence of periodic intervals $\left(J_{n}\right)_{n \in \mathbb{N}}$ with periods $\left(k_{n}\right)_{n \in \mathbb{N}}$ such that:

(1) $k_{1} \geq 1$, and $k_{n+1}$ is the multiple of $k_{n}$ for all $n \geq 1$;

(2) $J_{n+1} \subset J_{n}$, and every connected components of $\bigcup_{i=0}^{k_{n}-1} J_{n}$ contains the same numbers of the connected components of $\bigcup_{i=0}^{k_{n+1}-1} J_{n+1}$;

(3) $\omega(x, f) \subset \bigcap_{n \geq 1} \bigcup_{i=0}^{k_{n}-1} f^{i}\left(J_{n}\right)$ and $\omega(x, f)$ does not contain periodic points.

Lemma 3.24. Let $f \in C(M, M)$ with $h_{\text {top }}(f)=0$ and $\operatorname{Per}(f) \neq \emptyset$. Assume that $x \in M$ and $\omega(x, f)$ is infinite. Let $\left(J_{n}\right)_{n \in \mathbb{N}}$ and $\left(k_{n}\right)_{n \in \mathbb{N}}$ provided by Lemma 3.23 . Assume that $K$ is a periodic subinterval of $M$ with period $m$ and $\omega(x, f) \cap \bigcup_{i=0}^{m-1} f^{i}(K) \neq \emptyset$. Then there exists some $n \in \mathbb{N}$ such that $\bigcup_{i=0}^{k_{n}-1} f^{i}\left(J_{n}\right) \subset \bigcup_{i=0}^{m-1} f^{i}(K)$.

Proof. As $\left\{k_{n}\right\}$ is strictly increasing, $\bigcap_{n=1}^{\infty} \bigcup_{i=0}^{k_{n}-1} f^{i}\left(J_{n}\right)$ has uncountably many connected components and at most countably many of them can be non-degenerate. Moreover, each connected component intersects $\omega(x, f)$. By Lemma 3.22, $\omega(x, f) \subset \bigcup_{i=0}^{m-1} f^{i}(K)$. So there exists $i_{0} \in\{0,1, \ldots, m-1\}$ such that $f^{i_{0}}(K)$ contains at least three nondegenerate connected components of $\bigcap_{n=1}^{\infty} \bigcup_{i=0}^{k_{n}-1} f^{i}\left(J_{n}\right)$. Then there exist $n \in \mathbb{N}$ and $j_{0} \in\left\{0,1, \ldots, k_{n}-1\right\}$ such that $f^{j_{0}}\left(J_{n}\right) \subset f^{i_{0}}(K)$. As $J_{n}$ and $K$ are periodic, we have $\bigcup_{i=0}^{k_{n}-1} f^{i}\left(J_{n}\right) \subset \bigcup_{i=0}^{m-1} f^{i}(K)$.

3.4. Some auxiliary lemmas of circle maps. Our purpose is to study the properties of circle maps with zero topological entropy. At first of this subsection, we use the following lemmas to illustrate that we can restrict our study object to the circle maps with $|\operatorname{deg}(f)| \leq 1$, more precisely, non-extensible circle maps with $|\operatorname{deg}(f)| \leq 1$.

First we define extensible and non-extensible circle maps. This concept was first proposed by Mai in [18]. Here we modify the original definition in [18] according to what we need and describe as follow.

Definition 3.25. Let $f \in C(\mathbb{S}, \mathbb{S})$ and $F$ be a lifting of $f$. We call $f$ extensible if there exists $r \in \mathbb{R}$ and $n \in \mathbb{N}$ such that $\left|F^{n}([r, r+1])\right| \geq|\operatorname{deg}(f)|+1$. Otherwise it is called non-extensible.

In order to investigate the relationship between circle maps with zero entropy and the non-extensible circle maps with $|\operatorname{deg}(f)| \leq 1$, we introduce horseshoe and the relationship between horseshoe and positive entropy.

Definition 3.26. Let $f \in C(M, M)$. We say that two closed non-degenerate subintervals $K_{1}$ and $K_{2}$ of $M$ with disjoint interiors form a horseshoe if there exist some $n \in \mathbb{N}$ and subintervals $K_{i 1}$ and $K_{i 2}$ of $K_{i}$ such that $f^{n}\left(K_{i 1}\right)=K_{1}$ and $f^{n}\left(K_{i 2}\right)=K_{2}$ for $i=1,2$. When $M$ denote a non-degenerate closed interval $n=1$.

Lemma $3.27([12])$. Let $f \in C(M, M)$. If $f$ has a horseshoe then $h_{\text {top }}(f)>0$.

The following lemma tells us that a circle map with zero topological entropy is a nonextensible circle map with $|\operatorname{deg}(f)|<1$. It is an analogous result with Theorem 1.1 in [18] but still have some differences since the definitions of non-extensible are not the same. Here we give our proof. 
Lemma 3.28. Let $f \in C(\mathbb{S}, \mathbb{S})$ with $h_{\text {top }}(f)=0$. Then $f$ is a non-extensible circle map with $|\operatorname{deg}(f)| \leq 1$.

Proof. Let $F$ be a lifting of $f$. If $|\operatorname{deg}(f)| \geq 2$, then $|F([0,1])| \geq|\operatorname{deg}(f)| \geq 2$. Pick two closed subintervals $K_{1}$ and $K_{2}$ of $[0,1]$ with disjoint interiors such that $\left|F\left(K_{1}\right)\right|=$ $\left|F\left(K_{2}\right)\right|=1$, which implies that $e F\left(K_{1}\right)=e F\left(K_{2}\right)=\mathbb{S}$ and as a result $e\left(K_{1}\right)$ and $e\left(K_{2}\right)$ form a horseshoe of $f$. By Lemma 3.27, $h_{\text {top }}(f)>0$ which is a contradiction. Thus $|\operatorname{deg}(f)| \leq 1$.

Consider the case $|\operatorname{deg}(f)|=1$ and assume that $f$ is extensible. Then there exist $r \in \mathbb{R}$ and $n \in \mathbb{N}$ such that $\left|F^{n}([r, r+1])\right| \geq 2$. Analogous to the proof in the situation $|\operatorname{deg}(f)| \geq 2$, we can induce that $f$ has a horseshoe which is a contradiction. Thus $f$ is non-extensible.

Now we consider the case $\operatorname{deg}(f)=0$ and assume that $f$ is extensible. Note that in this case $F$ is a periodic function with period 1. Pick $x, y \in \mathbb{R}$ with $x<y<x+1$ such that $F(x)=\min F(\mathbb{R})$ and $F(y)=\max F(\mathbb{R})$. As $f$ is extensible, $F(y)-F(x) \geq 1$. Let $K_{1}=[x, y]$ and $K_{2}=[y, x+1]$. It is easy to see that $e\left(K_{1}\right)$ and $e\left(K_{2}\right)$ form a horseshoe of $f$ which is a contradiction. Thus $f$ is non-extensible.

For a circle map with zero topological entropy, we will be searching for a lifting $F$ and an interval $I$ such that $\left.F\right|_{I}$ become an interval map. The idea was first proposed in [18], here we restate the result and give our proof according to what we need.

Lemma $3.29([18])$. Let $f \in C(\mathbb{S}, \mathbb{S})$ with $h_{\text {top }}(f)=0$ and $\operatorname{Fix}(f) \neq \emptyset$. Then there exist a lifting $F$ of $f$ and a closed subinterval $I=[a, b]$ of $\mathbb{R}$ with $1 \leq b-a<2$ such that $F(I) \subset I$. Moreover,

(1) if $\operatorname{deg}(f)=0$, then $b-a=1$ and $|F([a, b])|<1$;

(2) if $\operatorname{deg}(f)=1$, then $F([a, b])=[a, b], F([a, b-1]) \subset[a, b-1]$ and $F([a+1, b]) \subset$ $[a+1, b]$

(3) if $\operatorname{deg}(f)=-1$, then $F([a, b])=[a, b], F([a, b-1]) \subset[a+1, b]$ and $F([a+1, b]) \subset$ $[a, b-1]$.

Proof. By Lemma 3.28, $|\operatorname{deg}(f)| \leq 1$ and $f$ is non-extensible. We divide the proof into three cases according to the degree of $f$.

Case 1: $\operatorname{deg}(f)=0$. Let $F$ be a lifting of $f$. As $F$ is a periodic function with period $1, F$ is bounded. As $f$ is non-extensible, $\max F(\mathbb{R})-\min F(\mathbb{R})<1$. Let $a=\min F(\mathbb{R})$. Then $F([a, a+1]) \subset[\min F(\mathbb{R}), \max F(\mathbb{R})] \subset[a, a+1]$.

Case 2: $\operatorname{deg}(f)=1$. Since $f$ has a fixed point, we can choose a lifting $F$ of $f$ such that $F$ has a fixed point $x$. Then $x+1$ is also a fixed point and $F([x, x+1]) \supset[x, x+1]$. Put $J=[x, x+1]$. By induction, $F^{n+1}(J) \supset F^{n}(J) \supset J$ for all $n \in \mathbb{N}$.

Let $K=\overline{\bigcup_{n=1}^{\infty} F^{n}(J)}$. It is clear that $K \supset J$ and $F(K)=K$. Since $f$ is non-extensible, for any $n \in \mathbb{N},\left|F^{n}(J)\right|<2$, hence $1 \leq|K| \leq 2$. Denote $K=[a, b]$. Since $F[a, b]=[a, b]$, $\left.\min F\right|_{[a, b-1]} \geq\left.\min F\right|_{[a, b]} \geq a$. If there exists some $y \in[a, b-1]$ such that $F(y)>b-1$, then $y+1 \in[a+1, b] \subset[a, b]$ and $F(y+1)=F(y)+1>b$, which contradicts to $F([a, b])=[a, b]$. Therefore for any $y \in[a, b-1], F(y) \leq b-1$. Thus we have $F([a, b-1]) \subset[a, b-1]$. Similarly, we have $F([a+1, b]) \subset[a+1, b]$.

If $|K|<2$, we take $I=K$. If $|K|=2$, that is $b-a=2$, then $b-1=a+1$. Since $F([a, b-1]) \subset[a, b-1], F([a+1, b]) \subset[a+1, b]$, we have $F(b-1)=b-1$ and 
$F(a+1)=a+1$. Thus $F(a)=a$ and so $F([a, b-1])=[a, b-1]$. In this case, it is enough to take $I=[a, a+1]$.

Case 3: $\operatorname{deg}(f)=-1$. Since $f$ has a fixed point, we can choose a lifting $F_{1}$ of $f$ such that $F_{1}$ has a fixed point $x$. Then $F_{1}(x+1)=F_{1}(x)-1=x-1$ and $F_{1}([x, x+1]) \supset[x-1, x]$. Now we choose another lifting $F=F_{1}+1$ of $f$ and put $J=[x, x+1]$. Then we have $F(J) \supset J$. By induction, $F^{n+1}(J) \supset F^{n}(J) \supset J$ for all $n \in \mathbb{N}$.

Let $K=\overline{\bigcup_{n=1}^{\infty} F^{n}(J)}$. It is clear that we have $K \supset J$ and $F(K)=K$. Since $f$ is non-extensible, $\left|F^{n}(J)\right|<2$ for all $n \in \mathbb{N}$ and $|K| \leq 2$. Denote $K=[a, b]$.

Since $F[a, b]=[a, b],\left.\max F\right|_{[a, b-1]} \leq b$. If there exists some $y \in[a, b-1]$ such that $F(y)<a+1$, then there exists $y+1 \in[a+1, b] \subset[a, b]$ such that $F(y+1)<a$, which is a contradiction to $F([a, b])=[a, b]$. Therefore $F([a, b-1]) \subset[a+1, b]$. Similarly, we have $F([a+1, b]) \subset[a, b-1]$.

If $|K|<2$, we take $I=K$. If $|K|=2$, that is $b-a=2$, then $b-1=a+1$. Since $F([a, a+1]) \subset[a+1, b]$ and $F([a+1, b]) \subset[a, a+1]$, we have $F(a+1)=a+1$ and $F(a)=F(a+1)+1=b$. It follows that $F([a, a+1])=[a+1, b]$. Now we choose a new lifting $F-1$ to replace $F$, then we have $(F-1)[a, b-1]=[a, b-1]$ which $|[a, b-1]|=1$. In this case, we take $I=[a, a+1]$.

Lemma 3.30 ([10]). Let $f \in C(\mathbb{S}, \mathbb{S})$ with $\operatorname{Fix}(f) \neq \emptyset$. If $F$ and $I$ are the lifting and interval provided by Lemma 3.29 then $e(\operatorname{Per}(F))=\operatorname{Per}(f)$.

The following lemma describes the $\omega$-limit set of $F$, which is the lifting of $f$ with $h_{\text {top }}(f)=0$ and $\operatorname{deg}(f)=1$.

Lemma 3.31. Let $f \in C(\mathbb{S}, \mathbb{S})$ with $h_{\text {top }}(f)=0, \operatorname{deg}(f)=1$ and $\operatorname{Fix}(f) \neq \emptyset$. Let $F$ and $I=[a, b]$ provided by Lemma 3.29 Then for any $x \in[a, b], \omega(x, F) \subset[a, b-1]$ or $\omega(x, F) \subset(b-1, a+1)$ or $\omega(x, F) \subset[a+1, b]$.

Proof. Since $F([a, b-1]) \subset[a, b-1]$ and $F([a+1, b]) \subset[a+1, b]$, if there exists some $n \in \mathbb{N}$ such that $F^{n}(x) \in[a, b-1]$ or $F^{n}(x) \in[a+1, b]$, then $\omega(x, F) \subset[a, b-1]$ or $\omega(x, F) \subset[a+1, b]$. Now we assume that for any $n \in \mathbb{N}, F^{n}(x) \in(b-1, a+1)$. Then $\omega(x, F) \subset[b-1, a+1]$. If $b-1 \in \omega(x, F)$, then $F(b-1) \in \omega(x, F) \subset[b-1, a+1]$. Since $F(b-1) \in F([a, b-1]) \subset[a, b-1], F(b-1)=b-1$. By Lemma3.21, $\omega(x, F)=\{b-1\}$ and then $\omega(x, F) \subset[a, b-1]$. Similarly, if $a+1 \in \omega(x, F)$ then $\omega(x, F)=\{a+1\} \subset[a+1, b]$. If $b-1, a+1 \notin \omega(x, F)$, then $\omega(x, F) \subset(b-1, a+1)$.

Lemma 3.32. Let $f \in C(\mathbb{S}, \mathbb{S})$ with $h_{\mathrm{top}}(f)=0, \operatorname{deg}(f)=1$ and $\operatorname{Fix}(f) \neq \emptyset$. Assume that $F$ and $I=[a, b]$ are the lifting and interval provided by 3.29. If $J$ is a periodic subinterval of I with period $n>1$, then exactly one of the following alternatives holds:

(1) $J \subset[a, b-1], \bigcup_{j=0}^{n-1} F^{j}(J) \subset[a, b-1]$;

(2) $J \subset[a+1, b], \bigcup_{j=0}^{n-1} F^{j}(J) \subset[a+1, b]$;

(3) $J \subset(b-1, a+1), \bigcup_{j=0}^{n-1} F^{j}(J) \subset(b-1, a+1)$.

Proof. Assume that $J \cap[a, b-1] \neq \emptyset$. If $J \cap(b-1, b] \neq \emptyset$, then $b-1 \in J$. Therefore for any $k \in \mathbb{N}, f^{k}(J) \cap[a, b-1] \neq \emptyset$. In particular, $f^{n-1}(J) \cap[a, b-1] \neq \emptyset$. Since $J \cap f^{n-1}(J)=\emptyset$, $b-1 \notin f^{n-1}(J)$, thus $f^{n-1}(J) \subset[a, b-1)$ and $f^{n}(J)=f\left(f^{n-1}(J)\right) \subset[a, b-1] \neq J$. Which is a contradiction since $J$ is a periodic interval with period $n$. Therefore $J \subset[a, b-1]$.

Similarly, if $J \cap[a+1, b] \neq \emptyset$, then $J \subset[a+1, b]$. 
Since $f^{i}(J)$ is a periodic interval with period $n$, by the above discussion we can get the conclusion.

Lemma 3.33. Let $f \in C(\mathbb{S}, \mathbb{S})$ with $h_{\text {top }}(f)=0, \operatorname{deg}(f)=1$ and $\operatorname{Fix}(f) \neq \emptyset$. Let $F$ and $I=[a, b]$ be a lifting and an interval provided by Lemma 3.29 If $K$ is a periodic interval of $\mathbb{S}$ with period $n>1$. Then there exists a periodic interval $J$ of I with period $n$ such that $e(J)=K$.

Proof. Since $K, f(K), \cdots, f^{n-1}(K)$ are mutually disjoint and $|K|<1$, there exists an interval $J \subset[a, a+1)$ or $J \subset(b-1, b]$ such that $e(J)=K$. Without loss of generality, we assume that $J \subset[a, a+1)$. Since $e \circ F^{i}=f^{i} \circ e, J, F(J), \cdots, F^{n-1}(J)$ are mutually disjoint and $e\left(F^{n}(J)\right)=f^{n}(e(J))=f^{n}(K)=K=e(J)$.

If $J \cap[a, b-1] \neq \emptyset$ and $J \cap(b-1, a+1) \neq \emptyset$, then $b-1 \in J$. Since $F([a, b-1]) \subset[a, b-1]$, for any $i, F^{i}(J) \cap[a, b-1] \neq \emptyset$ and in particular $F^{n-1}(J) \cap[a, b-1] \neq \emptyset$. Note that $J \cap F^{n-1}(J)=\emptyset, b-1 \notin F^{n-1}(J)$, and $F^{n-1}(J)$ is an interval, then $F^{n-1}(J) \subset[a, b-1)$. But $F([a, b-1]) \subset[a, b-1]$ and $F^{n}(J) \subset[a, b-1]$ which is a contradiction since $e\left(F^{n}(J)\right)=K=e(J)$. Therefore either $J \subset(b-1, a+1)$ or $J \subset[a, b-1]$.

Since both $\left.e\right|_{(b-1, a+1)}$ and $\left.e\right|_{[a, b-1]}$ are one to one, $F^{n}(J)=\left.e^{-1}\right|_{(b-1, a+1)}(K)=J$ and $F^{n}(J)=\left.e^{-1}\right|_{[a, b-1]}(K)=J$. In conclusion $J$ is a periodic interval with period $n$.

\section{THE EQUIVALENCE OF NON-SEPARABLE PAIRS, IT-PAIRS AND IN-PAIRS}

In this section we first introduce non-separable pairs for both interval maps and circle maps. Then we study the relationship between the non-separable pairs of $f$ and $f^{p}$ and the relationship between the non-separable pairs of $f$ and its lifting.

Definition 4.1. Let $f \in C(M, M)$. A pair $\langle x, y\rangle \in M \times M$ is called separable if there exist two periodic intervals $J_{1}$ and $J_{2}$ such that $J_{1} \cap J_{2}=\emptyset, x \in J_{1}$ and $y \in J_{2}$. A pair $\langle x, y\rangle \in M \times M$ with $x \neq y$ is called non-separable if there exists $z \in M$ such that $x, y \in \omega(z, f)$ and $\langle x, y\rangle$ is not separable. Denote by $N S(M, f)$ the set of all non-separable pairs of $f$.

Note that we require that the points in a non-separable pair are in the same $\omega$-limit set. We have the following characterization of non-separable pairs.

Lemma 4.2. Let $f \in C(M, M), h_{\mathrm{top}}(f)=0$ and $\operatorname{Per}(f) \neq \emptyset$. Assume that $x \neq y \in M$ and there exists $z \in M$ such that $x, y \in \omega(z, f)$. Then $\langle x, y\rangle$ is non-separable if and only if there exists a sequence of periodic intervals $\left\{K_{n}\right\}_{n=1}^{\infty}$ with periods $\left\{m_{n}\right\}_{n=1}^{\infty}$ such that $K_{n+1} \subset K_{n}, \lim _{n \rightarrow \infty} m_{n}=\infty$ and $x, y \in K_{n}$ for all $n \in \mathbb{N}$.

Proof. If $\omega(z, f)$ is finite, then it is a periodic orbit. Therefore $\langle x, y\rangle$ is separable and the result is clear. Now we consider the situation when $\omega(z, f)$ is infinite. Let $\left(J_{n}\right)_{n \in \mathbb{N}}$ and $\left(k_{n}\right)_{n \in \mathbb{N}}$ provided by Lemma 3.23 for the period portion of $\omega(z, f)$.

$(\Rightarrow)$ If $\langle x, y\rangle$ is non-separable, then for each $n \in \mathbb{N}$ there exists $i_{n} \in\left\{0,1, \ldots, k_{n}-1\right\}$ such that $x, y \in f^{i_{n}}\left(J_{n}\right)$. Thus $\left(J_{n}\right)_{n \in \mathbb{N}}$ and $\left(k_{n}\right)_{n \in \mathbb{N}}$ are as required.

$(\Leftarrow)$ Assume that $\langle x, y\rangle$ is separable, that is, there exist two periodic intervals $M_{1}$ and $M_{2}$ such that $M_{1} \cap M_{2}=\emptyset, x \in M_{1}$ and $y \in M_{2}$. Denote by $p_{1}$ and $p_{2}$ the period of $K_{1}$ and $K_{2}$ respectively. By Lemma 3.24, there exist $n_{1}, n_{2} \in \mathbb{N}$ such that $\bigcup_{i=0}^{k_{n_{1}}-1} f^{i}\left(J_{n_{1}}\right) \subset$ 
$\bigcup_{i=0}^{p_{1}-1} f^{i}\left(M_{1}\right)$ and $\bigcup_{i=0}^{k_{n_{2}}-1} f^{i}\left(J_{n_{2}}\right) \subset \bigcup_{i=0}^{p_{2}-1} f^{i}\left(M_{2}\right)$. Without loss of generality, we assume that $n_{1} \leq n_{2}$, then $J_{n_{1}} \supset J_{n_{2}}$. There exist unique $i_{1}, i_{2} \in\left\{0,1, \ldots, k_{n_{2}}-1\right\}$ such that $x \in f^{i_{1}}\left(J_{n_{2}}\right)$ and $y \in f^{i_{2}}\left(J_{n_{2}}\right)$. As $x \in M_{1}, y \in M_{2}$ and $M_{1} \cap M_{2}=\emptyset$, we have $i_{1} \neq i_{2}$. By Lemma 3.23, $x$ is not eventually periodic. For each $n \in \mathbb{N}$, there exists $j \in \mathbb{N}$ such that $f^{j}(x)$ are in the interior of $\bigcup_{i=0}^{m_{n}-1} f^{i}\left(K_{n}\right)$ and then $\omega(z, f) \subset \bigcup_{i=0}^{m_{n}-1} f^{i}\left(K_{n}\right)$. By Lemma 2.8(5) of [15], there exists $n_{3} \in \mathbb{N}$ such that $\bigcup_{i=0}^{m_{n_{3}}-1} f^{i}\left(K_{n_{3}}\right) \subset \bigcup_{i=0}^{k_{n_{2}}-1} f^{i}\left(J_{n_{2}}\right)$. Note that $x, y \in K_{n_{3}}$ and then there exists $i_{0} \in\left\{0,1, \ldots, k_{n_{2}}-1\right\}$ such that $x, y \in f^{i_{0}}\left(J_{n_{2}}\right)$. This is a contradiction.

Lemma 4.3. Let $f \in C(M, M), h_{\text {top }}(f)=0$ and $\operatorname{Per}(f) \neq \emptyset$. Then for $p \in \mathbb{N}$ which is the minimal period provided by Theorem $3.14 N S(M, f)=N S\left(M, f^{p}\right)$.

Proof. $(\Rightarrow)$ Let $\langle x, y\rangle \in N S(M, f)$. By the definition there exists $z \in M$ such that $x, y \in \omega(z, f)$. Let $\left(J_{n}\right)_{n \in \mathbb{N}}$ and $\left(k_{n}\right)_{n \in \mathbb{N}}$ provided by Lemma 3.23. For every $n \in \mathbb{N}$, there exists $i_{n} \in\left\{0,1, \ldots, k_{n}-1\right\}$ such that $x, y \in f^{i_{n}}\left(J_{n}\right)$. It is easy to see that for each $j \in\left\{0,1, \ldots, k_{n}-1\right\}, f^{j}\left(J_{n}\right)$ is a periodic interval of $f^{p}$ with period $\operatorname{lcm}\left(k_{n}, p\right) / p$. In particular, $f^{i_{n}}\left(J_{n}\right)$ is a periodic interval of $f^{p}$. Now we show that there exists $j_{0} \in \mathbb{N}$ such that $x, y \in \omega\left(f^{j_{0}}(z), f^{p}\right)$.

By Lemma 3.13, Lemma 3.14 and Remark 3.15, there exists some $n$ large enough such that $p$ divides $k_{n}$. Without loss of generality, $p$ dividess $k_{1}$. Denote $m=\frac{k_{1}}{p}$. If $x \in \omega\left(f^{i_{0}}(z), f^{p}\right), y \in \omega\left(f^{j_{0}}(z), f^{p}\right)$ where $i_{0} \neq j_{0}$, by Lemma 3.23, we can assume that $\omega\left(z, f^{k_{1}}\right) \subset J_{1}$. Then

$$
\omega\left(f^{i_{0}}(z), f^{p}\right)=\bigcup_{j=0}^{m-1} f^{i_{0}+j p}\left(\omega\left(z, f^{k_{1}}\right)\right) \subset \bigcup_{j=0}^{m-1} f^{i_{0}+j p}\left(J_{1}\right)
$$

and

$$
\omega\left(f^{j_{0}}(z), f^{p}\right)=\bigcup_{j=0}^{m-1} f^{j_{0}+j p}\left(\omega\left(z, f^{k_{1}}\right)\right) \subset \bigcup_{j=0}^{m-1} f^{j_{0}+j p}\left(J_{1}\right) .
$$

Therefore $\omega\left(f^{i_{0}}(z), f^{p}\right) \cap \omega\left(f^{j_{0}}(z), f^{p}\right)=\emptyset$, which is a contradiction since $x, y \in f^{i_{1}}\left(J_{1}\right)$. Thus there exists $j_{0} \in \mathbb{N}$ such that $x, y \in \omega\left(f^{j_{0}}(z), f^{p}\right)$.

We can get the conclusion that $\langle x, y\rangle$ is a non-separable pair of $\left(M, f^{p}\right)$.

$(\Leftarrow)$ Let $\langle x, y\rangle \in N S\left(M, f^{p}\right)$. By the definition there exists some $z \in \mathbb{S}$ such that $x, y \in \omega\left(z, f^{p}\right)$, therefore $x, y \in \omega(z, f)$. If $\langle x, y\rangle$ is separable for $f$, then there exist two periodic intervals $J_{1}$ and $J_{2}$ for $f$ such that $J_{1} \cap J_{2}=\emptyset, x \in J_{1}$ and $y \in J_{2}$. Note that $J_{1}$ and $J_{2}$ are also periodic intervals for $f^{p}$ and then $\langle x, y\rangle$ is separable for $f^{p}$. By Lemma 4.2, this is a contradiction. Thus $\langle x, y\rangle$ is not separable for $f$ and by lemma 4.2 again $\langle x, y\rangle \in N S(M, f)$.

The following lemma characterizes the relationship between the non-separable pairs of circle map and its lifting.

Lemma 4.4. Let $f \in C(\mathbb{S}, \mathbb{S}), h_{\mathrm{top}}(f)=0$ and $\operatorname{Fix}(f) \neq \emptyset$. Denote $F$ and I provided by the Lemma 3.29. Then $e \times e(N S(I, F))=N S(\mathbb{S}, f)$.

Proof. By Lemma 3.28, $|\operatorname{deg}(f)| \leq 1$ and $f$ is non-extensible. We divide the proof into three cases according to the degree of $f$. 
Case 1: $\operatorname{deg}(f)=0$. As $|I|=1$, it is easy to see that if $J$ is a periodic interval of $I$ with period $n \geq 2$, then $e(J)$ is also a periodic interval of $\mathbb{S}$ with period $n$. And if $K$ is a periodic interval of $\mathbb{S}$ with period $n \geq 2$, then we can pick a periodic interval $\widetilde{K}$ of $I$ such that $e(\widetilde{K})=K$. As $e$ is a homeomorphism when it is restricted to an interval with length less than 1 , by the definition of non-separable pairs, it is not hard to see that $e \times e(N S(I, F))=N S(\mathbb{S}, f)$.

Case 2: $\operatorname{deg}(f)=1$. Assume that $\langle x, y\rangle \in N S(I, F)$. Since $x, y \in \omega(z, F)$ for some $z \in I$, by Lemma 2.8, $e(x), e(y) \in \omega(e(z), f)$. By the definition, there exists a sequence of periodic intervals of $(I, F),\left\{J_{n}\right\}_{n=1}^{\infty}$ with periods $\left\{m_{n}\right\}_{n=1}^{\infty}$ such that $J_{n+1} \subset J_{n}$, $\lim _{n \rightarrow \infty} m_{n}=\infty$ and $x, y \in J_{n}$ for all $n \in \mathbb{N}$. Since $m_{n} \rightarrow \infty$, we can assume that $m_{n}>1$, by Lemma 3.32, for any $n \in \mathbb{N}, \bigcup_{j=0}^{m_{n}-1} F^{j}\left(J_{n}\right) \subset[a, b-1], \bigcup_{j=0}^{m_{n}-1} F^{j}\left(J_{n}\right) \subset[a+1, b]$, or $\bigcup_{j=0}^{m_{n}-1} F^{j}\left(J_{n}\right) \subset(b-1, a+1)$. Since $|[a, b-1]|<1,|[a+1, b]|<1$ and $|(b-1, a+1)| \leq 1$. Denote $J_{n}^{\prime}=e\left(J_{n}\right)$, it is obvious that $\left\{f^{j}\left(J_{n}^{\prime}\right)\right\}_{j=0}^{m_{n}-1}$ form a cycle of intervals of $\mathbb{S}$. Therefore there exists a sequence of periodic intervals of $(\mathbb{S}, f),\left\{J_{n}^{\prime}\right\}_{n=1}^{\infty}$ with periods $\left\{m_{n}\right\}_{n=1}^{\infty}$ such that $J_{n+1}^{\prime} \subset J_{n}^{\prime}, \lim _{n \rightarrow \infty} m_{n}=\infty$ and $e(x), e(y) \in J_{n}^{\prime}$ for all $n \in \mathbb{N}$. We conclude that $\langle e(x), e(y)\rangle$ is a non-separable pair of $(\mathbb{S}, f)$.

Now we assume that $\langle x, y\rangle \in N S(\mathbb{S}, f)$. By the definition, there exists $z \in \mathbb{S}$ such that $x, y \in \omega(z, f)$ and there exists a sequence of periodic intervals $\left\{J_{n}\right\}_{n=1}^{\infty}$ of $(\mathbb{S}, f)$ with period $\left\{m_{n}\right\}_{n=1}^{\infty}$ such that $J_{n+1} \subset J_{n}, \lim _{n \rightarrow \infty} m_{n}=\infty$ and for any $n \in \mathbb{N}, x, y \in J_{n}$. Since $m_{n} \rightarrow \infty$, we can assume that $m_{n}>1$. According to Lemma 3.32, for any $n$, there exists a periodic interval $J_{n}^{\prime}$ with period $m_{n}$ of $I$ such that $e\left(J_{n}^{\prime}\right)=J_{n}$. By lemma 3.32, $J_{n}^{\prime} \subset[a, b-1], J_{n}^{\prime} \subset(b-1, a+1)$ or $J_{n}^{\prime} \subset[a+1, b]$ for all $n$. By taking subsequence, we can assume that for any $n, J_{n}^{\prime} \subset[a, b-1]$. There exists some $k$ large enough such that $f^{k}(z) \in J_{1}$. Thus we can take $z^{\prime} \in J_{1}^{\prime}$ such that $e\left(z^{\prime}\right)=f^{k}(z)$ and as a result $e\left(\omega\left(z^{\prime}, F\right)\right)=\omega\left(f^{k}(z), f\right)=\omega(z, f)$. Taking $x^{\prime}, y^{\prime} \in J_{1}^{\prime}$ such that $e\left(x^{\prime}\right)=x$ and $e\left(y^{\prime}\right)=y$. Since $|b-1-a|<1$ and $e$ is a homeomorphism when it is restricted to an interval whose length is less than $1, x^{\prime}, y^{\prime} \in \omega\left(z^{\prime}, F\right)$ and $x^{\prime}, y^{\prime} \in J_{n}^{\prime}$. We get the conclusion that $\left\langle x^{\prime}, y^{\prime}\right\rangle$ is a non-separable pair of $(I, F)$.

Case 3: $\operatorname{deg}(f)=-1$. By Lemma 4.3, $N S(\mathbb{S}, f)=N S\left(\mathbb{S}, f^{2}\right)$ and $N S\left(I, F^{2}\right)=N S(I, F)$. Note that $\operatorname{deg}\left(f^{2}\right)=1$ and $F^{2}$ is a lifting of $f^{2}$. By Case $2, e \times e\left(N S\left(I, F^{2}\right)\right)=N S\left(\mathbb{S}, f^{2}\right)$. Thus, $e \times e(N S(I, F))=N S(\mathbb{S}, f)$.

We will need the following result for interval maps.

Theorem $4.5([13])$. Let $f \in C(I, I)$ and $h_{\text {top }}(f)=0$. Then $N S(I, f) \backslash \Delta=I T(I, f) \backslash \Delta=$ $I N(I, f) \backslash \Delta$.

Now we are ready to prove our first main result (Theorem 1.2). Using notations introduced in sections 2 and 3, we can restate it as follows.

Theorem 4.6. Let $f \in C(\mathbb{S}, \mathbb{S})$ and $h_{\mathrm{top}}(f)=0$. Then $N S(\mathbb{S}, f) \backslash \Delta=I T(\mathbb{S}, f) \backslash \Delta=$ $I N(\mathbb{S}, f) \backslash \Delta$.

Proof. Since $h_{\text {top }}(f)=0$, if $f$ has fixed point, by Lemma 3.29, we can find a lifting $F$ of $f$, such that there exists an interval $I$ which satisfys that $1 \leq|I|<2$ and $\left.F\right|_{I}$ is an interval map. By Lemma 3.9, $h_{\text {top }}\left(\left.F\right|_{I}\right) \leq h_{\text {top }}(f)=0$, that is $h_{\text {top }}\left(\left.F\right|_{I}\right)=0$. 
$N S(\mathbb{S}, f) \subset I N(\mathbb{S}, f):$ Assume that $\langle x, y\rangle \in N S(\mathbb{S}, f)$, by Lemma 4.4, there exists a non-separable pair $\left\langle x_{1}, y_{1}\right\rangle$ of $(I, F)$ such that $\pi\left(x_{1}\right)=x$ and $\pi\left(y_{1}\right)=y$. By Lemma 4.5, $\left\langle x_{1}, y_{1}\right\rangle$ is an IN-pair of $(I, F)$. And by Lemma 2.13, $\langle x, y\rangle$ is an IN-pair of $(\mathbb{S}, f)$.

$I N(\mathbb{S}, f) \subset I T(\mathbb{S}, f)$ : Assume that $\langle x, y\rangle \in N S(\mathbb{S}, f)$, by Lemma 2.13, there exists a IN-pair $\left\langle x_{1}, y_{1}\right\rangle$ of $(I, F)$ such that $\pi\left(x_{1}\right)=x$ and $\pi\left(y_{1}\right)=y$. By Lemma 4.5, $\left\langle x_{1}, y_{1}\right\rangle$ is an IT-pair of $(I, F)$. And by Lemma 2.13, $\langle x, y\rangle$ is an IT-pair of $(\mathbb{S}, f)$.

$I T(\mathbb{S}, f) \subset N S(\mathbb{S}, f)$ : Assume that $\langle x, y\rangle \in N S(\mathbb{S}, f)$, by Lemma 2.13, there exists a IT-pair $\left\langle x_{1}, y_{1}\right\rangle$ of $(I, F)$ such that $\pi\left(x_{1}\right)=x$ and $\pi\left(y_{1}\right)=y$. By lemma 4.5, $\left\langle x_{1}, y_{1}\right\rangle$ is an non-separable pair of $(I, F)$. And by Lemma $4.4\langle x, y\rangle$ is an non-separable pair of $(\mathbb{S}, f)$.

If $\operatorname{Per}(f) \neq \emptyset$, without loss of generallity we assume that $f$ has a periodic point whose period is $p$, by the proof of the situation when $\operatorname{Fix}(f) \neq \emptyset, N S\left(\mathbb{S}, f^{p}\right) \backslash \Delta=I T\left(\mathbb{S}, f^{p}\right) \backslash \Delta=$ $I N\left(\mathbb{S}, f^{p}\right) \backslash \Delta$. By Lemma4.3 and Lemma 2.14, $N S(\mathbb{S}, f) \backslash \Delta=I T(\mathbb{S}, f \backslash \Delta)=I N(\mathbb{S}, f) \backslash \Delta$.

If $\operatorname{Per}(f)=\emptyset$, by Lemma 3.19, $h_{\text {top }}^{\infty}(f)=0$, therefore $f$ has no IN-pairs. By the definition of IN-pair and IT-pair, $f$ has no IT-pairs. Besides, no periodic points implies no cycle of intervals which in turn implies that there is no non-separable pairs.

\section{MAXIMAL PATTERN ENTROPY AND TOPOLOGICAL NULL SYSTEMS}

In this section we first introduce maximal pattern entropy and some related concepts.

Definition 5.1. Let $(X, f)$ be a topological dynamical system and $\mathcal{U} \in C_{X}^{o}$. The maximal pattern entropy of $(X, f)$ with respect to $\mathcal{U}$ is defined as

$$
h_{\mathrm{top}}^{*}(f, \mathcal{U})=\lim _{n \rightarrow \infty} \frac{1}{n} \log \left(p_{X, \mathcal{U}}^{*}(n)\right)
$$

where

$$
p_{X, \mathcal{U}}^{*}(n)=\max _{\left(t_{1}, t_{2}, t_{3}, \cdots, t_{n}\right) \in \mathbb{Z}_{+}^{n}} N\left(\bigvee_{i=1}^{n} f^{-t_{i}} \mathcal{U}\right),
$$

and the maximal pattern entropy of $(X, f)$ is

$$
h_{\mathrm{top}}^{*}(f)=\sup _{\mathcal{U} \in C_{X}^{o}} h_{\mathrm{top}}^{*}(f, \mathcal{U}) .
$$

In [8], Huang and Ye showed that $h_{\text {top }}^{*}(f)=h_{\text {top }}^{\infty}(f)$.

Definition 5.2. Let $(X, f)$ be a topological dynamical system and $\mathcal{U} \in C_{X}^{0}$. We say that $p_{X, \mathcal{U}}^{*}$ is of polynomial order if there exists $C>0$ such that $p_{X, \mathcal{U}}^{*}(n) \leq C n^{C}$ for all $n \in \mathbb{N}$.

We first have the following observation.

Lemma 5.3. (1) Let $(X, f)$ be a topological dynamical system and $k \in \mathbb{N}$. Then $p_{X, \mathcal{U}}^{*}$ with respect to $f$ is of polynomial order for all $\mathcal{U} \in C_{X}^{o}$ if and only if so is with respect to $f^{k}$.

(2) Let $e:(X, f) \rightarrow(Y, g)$ be a factor map of two dynamical systems. If $p_{X, \mathcal{U}}^{*}$ is of polynomial order for all $\mathcal{U} \in C_{X}^{o}$ then $p_{Y, \mathcal{V}}^{*}$ is of polynomial order for all $\mathcal{V} \in C_{Y}^{o}$.

Proof. (1) Let $\mathcal{U} \in C_{X}^{o} \cdot p_{X, \mathcal{U}}^{*}$ with respect to $f$ and $f^{k}$ are denoted by $p_{X, \mathcal{U}, f}^{*}$ and $p_{X, \mathcal{U}, f^{k}}^{*}$ respectively. It is clear that $p_{X, \mathcal{U}, f^{k}}^{*}(n) \leq p_{X, \mathcal{U}, f}^{*}(k n)$ for all $n \in \mathbb{N}$. If $p_{X, \mathcal{U}, f}^{*}$ is of polynomial order, then so is $p_{X, \mathcal{U}, f^{k}}^{*}$. On the other hand, $p_{X, \mathcal{U}, f}^{*}(n) \leq p_{X, \bigvee_{i=0}^{k-1} f^{-i}(\mathcal{U}), f^{k}}(n)$ for all $n \in \mathbb{N}$. If $p_{X, \bigvee_{i=0}^{k-1} f^{-i}(\mathcal{U}), f^{k}}$ is of polynomial order, then so is $p_{X, \mathcal{U}, f^{*}}^{*}$ 
(2) Let $\mathcal{V} \in C_{Y}^{o}$. Then $\pi^{-1}(\mathcal{V}) \in C_{X}^{o}$. It is clear that $p_{Y, \mathcal{V}, g}^{*}(n) \leq p_{X, \pi^{-1}(\mathcal{V}), f}^{*}(n)$ for all $n \in \mathbb{N}$. If $p_{X, \pi^{-1}(\mathcal{V}), f}^{*}$ is of polynomial order, then so is $p_{Y, \mathcal{V}, g}^{*}$.

Remark 5.4. It is clear that if $p_{X, \mathcal{U}}^{*}$ is of polynomial order then $h_{\text {top }}^{*}(f, \mathcal{U})=0$. It is shown in [8] that if $\mathcal{U}$ is a clopen (i.e. closed and open) partition of $X$ then $h_{\text {top }}^{*}(f, \mathcal{U})=0$ implies that $p_{X, \mathcal{U}}^{*}(n)$ is of polynomial order. As a consequence, if $X$ is zero dimensional, then $(X, f)$ is null if and only if $p_{X, \mathcal{U}}^{*}(n)$ is of polynomial order for all $\mathcal{U} \in C_{X}^{o}$.

Definition 5.5. Let $(X, f)$ be a topological dynamical system and $A=\left\{a_{1}, a_{2}, \ldots\right\}$ be an increasing sequence of non-negative integers. For $\epsilon>0$, a subset $E$ of $X$ is called a $(A, n, \epsilon)$-spanning set if for any $x \in X$, there is some $y \in E$ such that $d\left(f^{a_{i}}(x), f^{a_{i}}(y)\right)<\epsilon$ for all $i \in 1,2, \ldots, n$. We define $r_{A}(n, \epsilon)$ as the minimal cardinality of $(A, n, \epsilon)$-spanning sets of $X$ and

$$
r^{*}(n, \epsilon)=\sup _{A} r_{A}(n, \epsilon),
$$

where the supreme ranges over all increasing sequences of non-negative integers.

We say that a subset $F$ is $(A, n, \epsilon)$-separated set of $X$ if for any $x, y \in F$, there is some $i \in\{1,2, \ldots, n\}$ such that $d\left(f^{a_{i}}(x), f^{a_{i}}(y)\right) \geq \epsilon$. We define $s_{A}(n, \epsilon)$ as the maximal cardinality of $(A, n, \epsilon)$-separated subsets of $X$ and

$$
s^{*}(n, \epsilon)=\sup _{A} s_{A}(n, \epsilon),
$$

where the supreme ranges over all increasing sequences of non-negative integers.

It is clear that $r_{A}(n, \epsilon) \leq s_{A}(n, \epsilon)$ and then $r^{*}(n, \epsilon) \leq s^{*}(n, \epsilon)$.

The following lemma is standard in topological dynamics, see e.g. [23, Theorem 7.7].

Lemma 5.6. Let $(X, f)$ be a topological dynamical system and $A=\left\{a_{1}, a_{2}, \ldots\right\}$ be an increasing sequence of non-negative integers.

(1) Let $\mathcal{U} \in C_{X}^{o}$ and $\delta$ be a Lebesgue number of $\mathcal{U}$. Then for every $n \in \mathbb{N}$,

$$
N\left(\bigvee_{i=1}^{n} f^{-t_{i}}(\mathcal{U})\right) \leq r_{A}\left(n, \frac{\delta}{2}\right) \leq s_{A}\left(n, \frac{\delta}{2}\right) .
$$

(2) Let $\epsilon>0$ and choose $\mathcal{V} \in C_{X}^{o}$ with a Lebesgue number less than $\epsilon$. Then

$$
r_{A}(n, \epsilon) \leq s_{A}(n, \epsilon) \leq N\left(\bigvee_{i=1}^{n} f^{-t_{i}}(\mathcal{V})\right) .
$$

By the definition of maximal pattern entropy and the above lemma, we have the following remark.

Remark 5.7. Let $(X, f)$ be a topological dynamical system. Then

$$
h_{\text {top }}^{*}(X, f)=\lim _{\epsilon \rightarrow 0} \limsup _{n \rightarrow \infty} \frac{1}{n} \log r^{*}(n, \epsilon)=\lim _{\epsilon \rightarrow 0} \limsup _{n \rightarrow \infty} \frac{1}{n} \log s^{*}(n, \epsilon),
$$

and the following statements are equivalent:

(1) $p_{X, \mathcal{U}}^{*}$ is of polynomial order for all $\mathcal{U} \in C_{X}^{o}$;

(2) $r^{*}(\cdot, \epsilon)$ is of polynomial order for all $\epsilon>0$;

(3) $s^{*}(\cdot, \epsilon)$ is of polynomial order for all $\epsilon>0$. 
Lemma 5.8. Let $f \in C(\mathbb{S}, \mathbb{S}), h_{\text {top }}(f)=0$ and $\operatorname{Fix}(f) \neq \emptyset$. Denote $F$ and I provided by Lemma 3.29 Then $(\mathbb{S}, f)$ is null if and only if $(I, F)$ is null.

Proof. $(\Leftarrow)$ If $(I, F)$ is null, by lemma 2.9 , $(\mathbb{S}, f)$ is null.

$(\Rightarrow)$ If $(\mathbb{S}, f)$ is null, by lemma 2.12, $f$ has no non-diagonal IN-pairs. By lemma 4.6, $f$ has no non-separable pairs. By lemma 4.4, $F$ has no non-separable pairs, thus $F$ is not Li-Yorke chaotic and by lemma $3.19 F$ is null.

We will also need the following result.

Theorem 5.9 ([13]). Let $f \in C(I, I)$. Then $f$ is null if and only iffor any $\mathcal{U} \in C_{X}^{o}, p_{X, \mathcal{U}}^{*}(n)$ is of polynomial order.

Now we are ready to prove our second main result.

Proof of Theorem 1.3 The sufficiency of the proof is clear, we only proof the necessity. First we assume that $\operatorname{Per}(f) \neq \emptyset$. Then there exists $p \in \mathbb{N}$ such that $\operatorname{Fix}\left(f^{p}\right) \neq \emptyset$. Let $F$ and $I$ provided by Lemma 3.29 for $f^{p}$. By Lemma 5.8, $(I, F)$ is null. Then by Lemma5.9, $p_{I, \mathcal{U}}^{*}(n)$ is of polynomial order for all $\mathcal{U} \in C_{I}^{o}$. As $e:(I, F) \rightarrow\left(\mathbb{S}, f^{p}\right)$ is a factor map, by Lemma 5.3 (2), $p_{\mathbb{S}, \mathcal{U}}^{*}$ with respect to $f^{p}$ is of polynomial order for all $\mathcal{U} \in C_{\mathbb{S}}^{o}$. Thus by Lemma $5.3(1), p_{\mathbb{S}, \mathcal{U}}^{*}$ with respect to $f$ is of polynomial order for all $\mathcal{U} \in C_{\mathbb{S}}^{o}$.

Now we consider the situation when $\operatorname{Per}(f)=\emptyset$. We divide the proof into two cases.

Case 1: $f$ is homeomorphism.

Fix $\epsilon>0$. Let $A=\left\{a_{1}, a_{2}, \cdots\right\}$ be a sequence of non-negative integers and $F=$ $\left\{x_{1}, x_{2}, \cdots, x_{k}\right\}$ be a $(A, n, \epsilon)$-separated set of $(\mathbb{S}, f)$. Without loss of generality, we assume that $x_{1}<x_{2}<\cdots<x_{k}<x_{1}$ under the ordering of $\mathbb{S}$ in Definition 3.3. Obviously in this case $f$ is an orientation preserving and $f^{a_{i}}\left(x_{1}\right)<f^{a_{i}}\left(x_{2}\right)<\cdots<f^{a_{i}}\left(x_{k}\right)<f^{a_{i}}\left(x_{1}\right)$ under the ordering of $\mathbb{S}$ for any $i \in\{1,2, \cdots, n\}$. Therefore for each $i$,

$$
\sum_{j=1}^{k-1} d\left(f^{a_{i}}\left(x_{j}\right), f^{a_{i}}\left(x_{j+1}\right)\right)+d\left(f^{a_{i}}\left(x_{k}\right), f^{a_{i}}\left(x_{1}\right)\right) \leq 1
$$

under the metric $d$ which is defined in Definition 3.2. Since $F=\left\{x_{1}, x_{2}, \cdots, x_{k}\right\}$ is $(A, n, \epsilon)$-separated, for any pair $\left\{x_{j}, x_{j+1}\right\}$ or $\left\{x_{k}, x_{1}\right\}$, where $j \in\{1,2, \cdots, k-1\}$, there exists some $i \in\{1,2, \cdots, n\}$ such that $d\left(f^{a_{i}}\left(x_{j}\right), f^{a_{i}}\left(x_{j+1}\right)\right)>\epsilon$, where $j \in\{1,2, \cdots, k\}$ and $x_{k+1}=x_{1}$. Therefore the number of the pairs $\left\{x_{j}, x_{j+1}\right\}$ cannot exceed $n\left(\left[\frac{1}{\epsilon}\right]+1\right)$ since if the number of the pairs exceeds $n\left(\left[\frac{1}{\epsilon}\right]+1\right)$, then there exists some $i$ such that the number of pairs $\left\{f^{a_{i}}\left(x_{j}\right), f^{a_{i}}\left(x_{j+1}\right)\right\}$ which satisfy that $d\left(f^{a_{i}}\left(x_{j}\right), f^{a_{i}}\left(x_{j+1}\right)\right)>\epsilon$ exceeds $\left[\frac{1}{\epsilon}\right]+1$, thus

$$
\sum_{j=1}^{k-1} d\left(f^{a_{i}}\left(x_{j}\right), f^{a_{i}}\left(x_{j+1}\right)\right)+d\left(f^{a_{i}}\left(x_{k}\right), f^{a_{i}}\left(x_{1}\right)\right)>1,
$$

which is a contradiction. Therefore, $k \leq n\left(\left[\frac{1}{\epsilon}\right]+1\right)$ and then $s_{A}(n, \epsilon) \leq n\left(\left[\frac{1}{\epsilon}\right]+1\right)$ since $F$ is arbitrary. Moreover, $s^{*}(n, \epsilon) \leq n\left(\left[\frac{1}{\epsilon}\right]+1\right)$ since the upper bound $n\left(\left[\frac{1}{\epsilon}\right]+1\right)$ does not depend on the sequence $A$, that is $s^{*}(\cdot, \epsilon)$ is of polynomial order. As $\epsilon$ is arbitrary, by Proposition 5.7, $p_{\mathbb{S}, \mathcal{U}}^{*}$ is of polynomial order for all $\mathcal{U} \in C_{\mathbb{S}}^{o}$.

Case 2: $f$ is not homeomorphism. 
By Lemma 3.18, $W=\omega(x, f)$ is the only $\omega$-limit set of $\mathbb{S}$ and it is a nowhere dense perfect set. Moreover, all the contiguous intervals are wandering and the image of any contiguous interval is either a contiguous interval or a point from $W$. By the properties of $\omega$-limit set, $f(W)=W$.

Fix $\epsilon>0$. Let $A=\left\{a_{1}, a_{2}, \cdots\right\}$ be a sequence of non-negative integers, $I_{1}, I_{2}, \cdots, I_{k}$ be all contiguous intervals longer than $\frac{\epsilon}{2}$ and $E_{W}$ be a $\left(A,\left.f\right|_{W}, n, \frac{\epsilon}{2}\right)$-spanning set.

First we consider the points whose $(A, f, n)$-trajectory is disjoint from $\bigcup_{i=1}^{k} I_{i}$ and we take $x$ as such a point. If $x \in W$ then $x$ is $(A, f, n, \epsilon)$-spanned by $E_{W}$. If $x \notin W$ we put $y$ to be an endpoint of the contiguous interval which contains $x$. Then $d\left(f^{a_{i}}(x), f^{a_{i}}(y)\right) \leq \frac{\epsilon}{2}$ for all $1 \leq i \leq n$ since the $(A, f, n)$-trajectory of $x$ is in $\mathbb{S} \backslash \bigcup_{i=1}^{k} I_{i}$. Since $y \in W$, there is a point $z \in E_{W}$ such that $y$ is $(A, f, n, \epsilon)$-spanned by $z$, as a conclusion the set $E_{W}$ $(A, f, n, \epsilon)$-spans all such points $x$.

Now we consider the points whose trajectory meet $\bigcup_{i=1}^{k} I_{i}$. Fix $N \in \mathbb{N}$ such that $N>\left[\frac{1}{\epsilon}\right]$. For $1 \leq i \leq n$ and $1 \leq j \leq k$, denote $I(i, j)=\left\{x: f^{a_{i}}(x) \in I_{j}\right\}$. It is obvious that $I(i, j)$ is a contiguous interval. Consider its $(A, f, n)$-trajectory $\left\{f^{a_{1}}(I(i, j)), f^{a_{2}}(I(i, j)), \cdots, f^{a_{n}}(I(i, j))\right\}$. Each elements in this trajectory is either a contiguous interval or a point from $W$ and all the elements are pairwise disjoint since $I(i, j)$ is wandering. At most $k$ of them have lengths greater than or equal to $\epsilon$ since $\left\{I_{i}\right\}_{i=1}^{k}$ are all the contiguous intervals longer than $\frac{\epsilon}{2}$. Cut each of such elements to $N$ segments shorter than $\epsilon$. All of the other elements of the trajectory will be consider to be segments themselves. To each $x \in I(i, j)$ assign its code-the $n$-tuple $\left(S_{1}(x), S_{2}(x), \cdots, S_{n}(x)\right)$ where $S_{i}(x)$ is the segment containing $f^{a_{i}}(x)$. We have at most $N^{k}$ different codes and all points with the same code can be $(A, f, n, \epsilon)$-spanned by one point. Therefore there is a set with cardinality at most $N^{k}$ which $(A, f, n, \epsilon)$-spans $I(i, j)$.

By Lemma 3.18, $\left.f\right|_{W}$ is an orientation preserving, moreover by linear extension of $\left.f\right|_{W}$ we obtain a homeomorphism $g \in C(\mathbb{S}, \mathbb{S})$. By the proof of Case 1 when $f$ is a homeomorphism, we have

$$
r\left(\left.f\right|_{W}, A, n, \epsilon / 2\right)=r\left(\left.g\right|_{W}, A, n, \epsilon / 2\right) \leq r(g, A, n, \epsilon / 2) \leq s(g, A, n, \epsilon / 2) \leq n\left[\frac{2}{\epsilon}\right],
$$

therefore $r(f, A, n, \epsilon) \leq r\left(\left.f\right|_{W}, A, n, \epsilon / 2\right)+n \cdot k \cdot N^{k} \leq n\left[\frac{2}{\epsilon}\right]+n \cdot k \cdot N^{k}$. Moreover, since $N$ and $k$ are independent of the sequence $A$,

$$
r^{*}(f, n, \epsilon)=\sup _{A} r(f, A, n, \epsilon) \leq n\left[\frac{1}{\epsilon}\right]+n k N^{k} .
$$

By Proposition 5.7, $p_{\mathbb{S}, \mathcal{U}}^{*}$ is of polynomial order for each open cover $\mathcal{U}$.

\section{ACKNOWLEDGEMENTS}

The author would like to thank Prof. Jian Li and Prof. Piotr Oprocha for the careful reading and helpful suggestions. This work was supported in part by the National Natural Science Foundation of China (11771264) and Guangdong Natural Science Foundation (2018B030306024). 


\section{REFERENCES}

[1] L. Alseda, J. Llibre, M. Misiurewicz, Combinatorial dynamics and entropy in dimension one, Second edition. Advanced Series in Nonlinear Dynamics, 5. World Scientific Publishing Co., Inc., River Edge, NJ, 2000.

[2] J. Auslander, Y. Katznelson, Continuous maps of the circle without periodic points, Israel J. Math. 32 (1979), no. 4, 375-381.

[3] A. M. Blokh, Dynamical systems on one-dimensional branched manifolds I (Russian), Teor. Funktsiř Funktsional. Anal. i Prilozhen. No. 46 (1986), 8-18; translation in J. Soviet Math. 48 (1990), no. 5, 500-508.

[4] N. Franzova, J. Smital, Positive topological sequence entropy characterizes chaotic maps, Proc. Amer. Math. Soc. 112 (1991), no. 4, 1083-1086.

[5] E. Glasner, X. Ye, Local entropy theory, Ergodic Theory Dynam. Systems 29 (2009), no. 2, 321-356.

[6] B. Hasselblatt, A. Katok, A First Course in Dynamics: With a Panorama of Recent Developments, Cambridge University Press, New York, 2003.

[7] R. Hric, Topological sequence entropy for the maps of the circle, Comment. Math. Univ. Carolin. 41 (2000), no. 1, 53-59.

[8] W. Huang, X. Ye, Combinatorial lemmas and applications to dynamics, Adv. Math. 220 (2009), no. 6, 1689-1716.

[9] D. Kerr, H. Li, Independence in topological and $C^{*}$-dynamics, Math. Ann. 338 (2007), 869-926.

[10] M. Kuchta, Characterization of chaos for continuous maps of the circle, Comment. Math. Univ. Carolin. 31 (1990), no. 2, 383-390.

[11] M. Kuchta, J. Smital, Two-point scrambled set implies chaos, European Conference on Iteration Theory (Caldes de Malavella, 1987), 427-430, World Sci. Publ., Teaneck, NJ, 1989.

[12] J. Llibre, M. Misiurewicz, Horseshoes, entropy and periods for graph maps, Topology 32 (1993), no. 3, 649-664.

[13] J. Li, Chaos and Entropy for Interval Maps, J. Dyn. Differ. Equ. 23 (2011), 333-352.

[14] J. Li, P. Oprocha, Y. Yang, T. Zeng, On dynamics of graph maps with zero topological entropy, Nonlinearity 30 (2017), no. 12, 4260-4276.

[15] J. Li, P. Oprocha, G. Zhang, On dynamics of quasi-graph maps, preprint, arXiv:1809.05617.

[16] J. Li, X. Ye, Recent development of chaos theory in topological dynamics, Acta Math. Sin. (Engl. Ser.) 32 (2016), no. 1, 83-114.

[17] T. Li, J. Yorke, Period three implies chaos, Am. Math. Monthly 82 (1975), no. 10, 985-992.

[18] J. Mai, Some dynamical system properties of self-maps of the circle and conditions equivalent to them, Adv. in Math. (China) 26 (1997), no. 3, 193-210.

[19] S. Ruette, Chaos on the interval, University Lecture Series, 67. American Mathematical Society, Providence, RI, 2017.

[20] S. Ruette, L. Snoha, For graph maps, one scrambled pair implies Li-Yorke chaos, Proc. Amer. Math. Soc. 142 (2014), no. 6, 2087-2100.

[21] J. Smital, Chaotic functions with zero topological entropy, Trans. Amer. Math. Soc. 297 (1986), no. 1, 269-282.

[22] F. Tan, X. Ye and R. Zhang, The set of sequence entropies for a given space, Nonlinearity, 23 (2010), 159-178.

[23] P. Walters, An introduction to ergodic theory, Graduate Texts in Mathematics, 79(1982). SpringerVerlag, New York-Berlin.

Department of Mathematics, Shantou University, Shantou, Guangdong, 515063, China

E-mail address: ynyangchs@foxmail.com 